\title{
ARTIGOS
}

\section{PROPOSTA DE EXPANSÃO PARA A CIDADE DE NOVA ESPERANÇA-PR}

\section{PROPOSED EXPANSION FOR CITY NOVA ESPERANÇA-PR}

\author{
MURILO PEREIRA TREVISAN | UNICESUMAR \\ PAULO ROBERTO NINO JÚNIOR | UNICESUMAR
}

\begin{abstract}
RESUMO
As pequenas e médias cidades brasileiras tem enfrentado um sério problema com o seu crescimento, que é a falta de planejamento urbano, permitindo assim acontecer uma expansão desordenada. Este projeto de pesquisa tem seu estudo de caso focado em avaliar a expansão da cidade de Nova Esperança através de levantamento de áreas habitadas e áreas a futura habitação, planejando locar áreas de indústrias e comercio, seguindo modelos positivos já existentes em outras pequenas e medias cidades por fim uma proposta de planejamento urbano para a cidade com mapeamento e zoneamento de forma a favorecer seu crescimento e atrair investimentos ao local.
\end{abstract}

PALAVRAS CHAVE: Urbanização; Planejamento; Zoneamento

\begin{abstract}
Small and medium-sized Brazilian cities have faced a serious problem with their growth, which is the lack of urban planning, allowing a disorderly expansion happen. This research project has its case study focused on evaluating the expansion of the city of Nova Esperança through a survey of inhabited areas and areas for future housing, planning to lease areas of industry and commerce, having as example existing positive models in other small and medium cities finally a proposal of urban planning for the city with mapping and zoning in order to promote growth and attract investment to the site.
\end{abstract}

KEY WORDS: Urbanization; Planning; Zoning 


\section{INTRODUÇÃO}

A urbanização no Brasil se difundiu de modo distinto dos países desenvolvidos, os países de primeiro mundo tiveram seu processo de urbanização correndo em torno de 100 a 200 anos, isso após a revolução industrial, no caso do Brasil a urbanização foi acelerada junto a ao processo de industrialização que durou em torno de 50 anos. Este crescimento urbano elevado se consolidou pelo fato do aumento da taxa de natalidade em nosso país, ocasionando assim uma urbanização não planejada. (RAIA JUNIOR, 1995). O termo planejamento urbano tem seu conceito muito além da semântica, pois, tem grande correlação com desenho urbano, gestão urbana e urbanismo, assim o planejamento urbano foi ganhando espaço no Brasil com a aprovação da Constituição de 1988, no qual os municípios receberam autonomia sobre sua expansão no território nacional. Atualmente apresentamos o termo planejamento urbano dando ênfase que é necessário um grande conjunto de medidas para se atingir um bom resultado. Desde modo ousamos dizer que planejar é buscar tendências e espaços para o desenvolvimento do projeto, porém, mais que necessário, uma obrigação, é obedecer às regras e leis, de uso e ocupação de solo, delimitando e criando estratégias para afim de manter e aumentar meIhor qualidade de vida dos munícipes. (DUARTE, 2007).

Ao se planejar uma cidade um bairro, ou até mesmo uma praça, é necessário saber muito bem o "para que" está sendo feito, encontra-se grandes projetos que não saem do papel por não ter fundamento, até mesmo construções abandonadas por não terem utilidade. Sendo assim, antes de se começar um projeto é importante saber da sua necessidade e futura utilidade. Desta forma, propõe-se um planejamento de expansão da cidade de Nova Esperança, tendo em vista o crescimento da cidade por estar em uma localização privilegiada por rodovias. No contexto urbano que cidade de Nova Esperança vive, desperta a necessidade de um projeto de planejamento urbano delimitando futuras áreas residências, comerciais e industriais, afim de inibir a desordem urbana.

Segundo SILVA (1993) grande parte das cidades Brasileiras de pequeno e médio porte são frutos de um processo de colonização nacional, seus planejamentos urbanos são por muitas vezes parecidos, utilizando-se do traçado denominado tipo grelha, de onde parte de um ponto central, que por vezes é a igreja matriz ou estação rodoviária ou ferroviária, partindo este ponto desenvolve-se pequenos comércios, várias ruas, entretanto poucas vias arteriais. A cidade de Nova Esperança, proposito e estudo deste trabalho, é exemplo deste modo de cidade.
Ao final deste trabalho espera-se mapear as áreas a serem ocupadas no futuro de forma organizada, prevendo maior crescimento físico financeiro a cidade. No momento presente a cidade se apresenta adequada a este estudo e planejamento, onde tem grandes chances de crescimento nos próximos anos, em que a população brasileira tem deixado os grandes centros a procura de pequenas cidades para melhor bem-estar e aumento da qualidade de vida. (LIMA, 1998)

Tem-se em objetivo desenvolver uma análise da cidade de Nova Esperança-PR e mapeamento de áreas não habitadas e mancha urbana; afim de estudar a geometrias das vias principais de acesso; analisar a infraestrutura disponível na cidade; e por fim destacar as áreas possíveis para industrias, residências.

\section{DESENVOLVIMENTO URBANO}

Dentro de um bom desenvolvimento do planejar de uma cidade, o termo zoneamento é valoroso. Neste caso, uma área urbana divide-se em setores destinados a certas atividades. Mantendo uma coerência e ordem no município, onde prevê melhor qualidade no bem-estar dos que utilizam e vivem na cidade ordenada. (PAIXÃO, 2013).

Zoneamento é um termo da área de urbanismo, que compreende em dividir em zonas, o termo foi criado na Alemanha no século XIX, porem ficou conhecido mundialmente após os Estados Unidos utilizar anos depois, o zoneamento era usado para dividir espaços e delimitar divisas. No Brasil, deu início a sua utilização na cidade do Rio de Janeiro na reforma urbanista Pereira Passos, deste em diante se difundiu pelo país, sendo de grande utilização nas cidades brasileiras desde as de pequeno porte até as grandes metrópoles. É grande sua importância em dividir em partes para prever crescimentos ordenados nas cidades. (BORGES, 2007)

\section{METODOLOGIA}

Com o intuito de obter êxito na pesquisa e no planejamento urbano futuro do município, utilizou-se de alguns métodos, são eles:

Em primeiro momento foi coletado dados junto a prefeitura da cidade, como, mapas, levantamentos cadastrais, demarcando as áreas não habitadas na cidade e delimitação da mancha urbana. Feito isto é analisado o mapa digital da cidade junto a outras informações coletadas in loco, dando sequência a proposta de expansão.

O estudo da geometria das vias foi feito através do mapeamento previamente disponibilizado pela prefeitura e em casos críticos levantamento in loco com trena. 
Deste modo deu-se sequência a marcar no mapa digital as vias principais da cidade que favorecem o acesso a que é necessário.

Junto a Companhia de Água e Companhia de Energia Elétrica foi coletado dados afim de analisar a estrutura hidráulica e elétrica dos pontos de expansão da cidade. Com o intuito de propor um planejamento economicamente viável. Realizou-se o mapeamento digital com uso do software AutoCAD e CorelDraw. Após os estudos de casos similares de outros municípios apresenta-se uma nova proposta de expansão de possíveis áreas industriais, residenciais, destacando por zonas de utilização.

\section{RESULTADOS}

\subsection{Caracterização geográfica}

O município de Nova Esperança é pertencente ao estado do Paraná na região noroeste e território Brasileiro na região sul, com uma superfície territorial total de $401,587 \mathrm{~km}^{2}$, altitude estima de 550 metros.

De acordo com o censo realizado pelo IBGE (Instituto Brasileiro de Geografia e Estatística) no ano de 2010 a população total deste município é de 26.615 habitantes, destes sendo 22.585 habitantes na zona urbana e 4.144 habitantes na zona rural.

A cidade de Nova Esperança é de fácil acesso, tendo ligações com as seguintes rodovias, BR-376, PR-218, PR463 e PR-935.

\subsection{Análise do cenário atual}

Com o passar dos anos a cidade de Nova Esperança teve seu crescimento radial em relação a parte comercial e central da cidade, modelo comum no cenário das pequenas cidades Brasileiras.

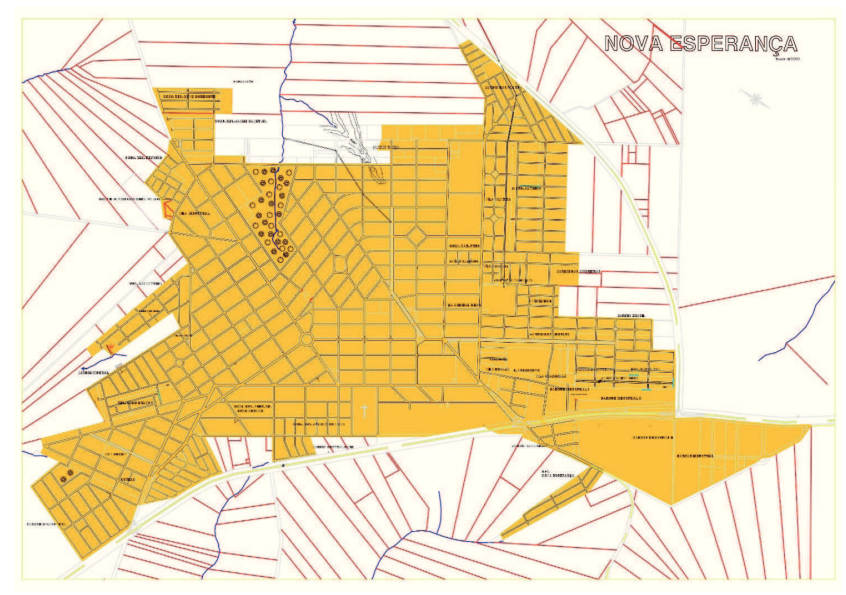

Figura 01: Mancha urbana de Nova Esperança-PR Fonte: Prefeitura municipal de Nova Esperança, 0 autor.
Em análise a mancha urbana da cidade de Nova Esperança (Figura 01) pode-se observar que as rodovias de acesso margeiam a cidade, favorecendo um fácil acesso, porem cria um limite de crescimento para a pequena cidade.

A cidade de Nova Esperança se enquadra no caso típico brasileiro onde a concentração da população é urbana e ultrapassa os $80 \%$ dos munícipes. O brasil tem grandes manchas urbanas, caso que se compreende a países desenvolvidos.

O aumento nessa mancha urbana, se dá com a imigração da zona rural para o centro das cidades, e essa concentração de pessoas na área urbana tende a crescer progressivamente junto o crescimento da população mundial.

Atualmente a cidade de Nova Esperança tem sido considerada uma cidade dormitório, pois parte da população tem sua geração de renda e seus maiores gastos em cidades maiores vizinhas, como, Maringá á $40 \mathrm{~km}$ e a cidade de Paranavaí com distância de $35 \mathrm{~km}$. Criando um déficit na economia municipal, e inviabilizando investimentos públicos, para o restante da população.

A cidade em estudo tem deficiência que se fala em transporte público, pois o serviço oferecido é somente de transporte intermunicipal e interestados. Fator relevante ao caso, são vias mal projetadas dificultando muitas vezes o acesso de veículos de maior porte nos bairros mais afastados.

Por se tratar de uma cidade de pequeno porte e baixa movimentação financeira as receitas públicas são de baixo nível, deixando precário o sistema de saúde pública e segurança.

\subsection{Análise do cenário álvo}

Com base nos levantamentos e estudos acima citados tem-se uma proposta de expansão para a cidade de Nova EsperançaPR. Levando em conta a qualidade de vida, orçamento financeiro, que estabeleceremos como sócio econômico.

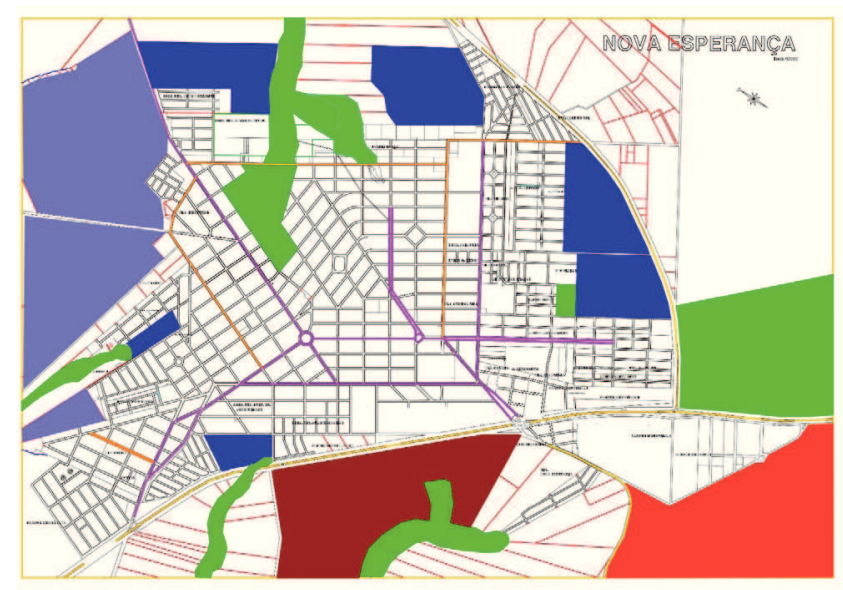

Figura 02: Zonas de expansão de Nova Esperança-PR Fonte: Prefeitura municipal de Nova Esperança, 0 autor. 
O mapa apresentado (figura 02) traz a cidade de Nova Esperança com uma proposta de expansão quanto a parte residencial e industrial, visando melhorias no âmbito sócio econômico do município.

\begin{tabular}{|l|c|}
\hline \multicolumn{2}{|c|}{ ÁREA DE EXPANÇÃO } \\
\hline Código & $\begin{array}{c}\text { Especificações de preservação } \\
\text { ambiental }\end{array}$ \\
\hline & $\begin{array}{c}\text { Área para fins residencias a } \\
\text { curto prazo }\end{array}$ \\
\hline & $\begin{array}{c}\text { Área para fins residencias a } \\
\text { longo prazo }\end{array}$ \\
\hline & $\begin{array}{c}\text { Área para fins industriais a } \\
\text { curto prazo }\end{array}$ \\
\hline & $\begin{array}{c}\text { Área para fins industriais a } \\
\text { longo prazo }\end{array}$ \\
\hline & $\begin{array}{c}\text { Ruas/ Avenidas de principais } \\
\text { acesso e comercio }\end{array}$ \\
\hline & Ruas/ Avenidas de acesso e \\
& comercio secundários \\
\hline & \\
\hline & \begin{tabular}{c} 
Rodovias de acesso \\
\hline
\end{tabular} \\
\hline
\end{tabular}

Tabela 01: Códigos de Zonas de expansão de Nova Esperança-PR Fonte: 0 autor (2016)

\subsection{1 Área de preservação ambiental}

Área de preservação ambiental é fato importante no processo de planejamento territorial, diversos órgãos públicos cobram o assunto, ainda maior é a cobrança quando se trata de expansão urbana. No mapa de proposta apresentado (figura 02) destacasse essas áreas afim de contribuir com o meio ambiente e ter um crescimento da cidade ordenado com leis vigentes em nosso país. Mantendo reservas legais, bem como proteção dos leitos de rios e córregos próximo a área urbana do município.

\subsection{2 Área residenciais}

Pertencente a proposta de expansão da cidade de Nova Esperança estão as áreas residências, com foco no bem-estar da população. Ás áreas residências foram dividias em dois grandes grupos, áreas para fins residências a curto prazo e áreas para fins residências a longo prazo.

Ás áreas residências a curto prazo foram considerado áreas não habitadas que estão próximas a bairros já estruturados, e com fácil acesso, é destacável neste grupo facilidade de implantação de sistema de energia elétrica, agua tratada e sistema de esgoto sanitário, bem como coleta de aguas pluviais e coleta de lixo. Estás áreas são de grande interesse por necessitarem de menor investimento financeiro para a implantação de loteamentos residenciais e estarem localizadas mais próximas a comercio de produtos e serviços.

Por tratar de uma proposta de expansão tem-se o conhecimento de necessidade de vários anos para atingir o objetivo, com este conhecimento, foi destacado a área residencial a longo prazo. Nestas terras foi levado em consideração a obrigação de maiores investimentos financeiros para se tornar adequada a moradia humana, estas áreas são mais afastadas do centro da cidade ficando com déficit de comercio e como a locomoção fica dificultada. Acesso a este grupo é precário, necessita-se de investimentos públicos e privados para progredir, com referência a analise atual do município citada anteriormente torna-se inviável utilização dessas áreas residências no âmbito presente, por estes fatores englobam-se em áreas a logo prazo.

\subsection{3 Áreas industriais}

As indústrias bem como o comercio de produtos e serviços são de grande importância para a cidade e todo o município, pois contribuem com a geração de renda aos colaboradores e recolhimento de impostos por parte do governo público.

Destacamos na proposta de expansão duas áreas industriais uma delas é área próxima ao atual parque industrial da cidade, essa área é de considerável importância por ter fácil acesso, e infraestrutura voltada a indústria e incentivos públicos, assim foi destacada como área industrial de curto prazo. Visando um grande crescimento na cidade Nova Esperança foi proposto um outro espaço voltado as indústrias, com maior espaço físico e fácil acesso de rodovia, o outro parque industrial necessita de grandes investimentos públicos ou privados, por isso foi considerado longo prazo para essa implantação na expansão da cidade. 
Um dos principais fatores a se criar uma área industrial é o acesso e disponibilidade de vias, para que recebam matéria prima e após exportem seus produtos. Neste sentido a cidade de Nova Esperança é favorecida por ter boas rodovias de acesso, tendo como principal a BR-376, onde margeia as áreas industrias da cidade.

\subsubsection{Sistema viário}

$\mathrm{O}$ acesso de produtos e serviços bem como locomoção de pessoas, é através do sistema viário, que possibilita o direito de ir e vir de cada brasileiro.

A cidade de Nova Esperança é alimentada por boas rodovias e de fácil acesso. Suas vias urbanas são destacadas na figura 02 como vias principais e secundarias. Nestas são de grande concentração o comercio e serviços públicos, tais como, bancos, mercados, escolas, hospitais, prefeitura.

As vias principais ligam os bairros e garantem a qualidade de vida dos munícipes, a proposta de expansão da cidade considera grandiosamente estas vias, pois precisam ainda garantir a locomoção de pessoas e produtos mesmo após o aumento de frota.

\section{CONCLUSÃO}

Levando-se em consideração o âmbito atual de Nova Esperança foi realizado um mapeamento da mancha urbana da cidade, visando esclarecer áreas habitadas e não habitadas, afim de poder preparar uma futura utilização para os espaços vazios.

O destaque das vias de acesso principal e secundários colaborou com a análise de acesso aos futuros loteamentos residências e concluímos a viabilidade dos espaços.

Percebemos que em grande parte da cidade é necessário pouco investimento para a expansão, porem salvo alguns locais que tem a necessidade de investimentos públicos como incentivo.

Somos levados a acreditar no potencial da cidade em estudo por sua boa localização, favorecendo a criação dos espaços destinados a industrias, separados da área residencial. Fato incomum na maioria das cidades de pequeno porte.

É ressaltado que a viabilidade deste projeto de expansão é condizente com a atualidade do município, porém são velozes as transformações dos espaços e ambientes das cidades, assim se faz necessário atualizações antes de pôr em prática, compreendendo sempre a estrutura social e econômica e aspectos físicos do local.

\section{REFERÊNCIAS}

DUARTE, Fabio. Planejamento Urbano. 2. ed. Curitiba: Ibpex, 2011. 177 p.

GOITIA, F. C. Breve História do Urbanismo. Editorial Presença. Portugal, 1982.

LIMA, Renato da Silva. EXPANSÃO URBANA E ACESSIBILIDADE - O CASO DAS CIDADES MÉDIAS BRASILEIRAS.1998. 91 f. Monografia (Especialização) - Curso de Engenharia Civil, Departamento de Transportes, Universidade de São Paulo Escola de Engenharia de São Carlos, São Carlos, SP, 1998. Cap. 2.2.

PAIXÃO, Maria José Pimentel da; AIALA, Carla Patricia Marrafon. PLANEJAMENTO URBANO: IMPORTANCIA DO ZONEMAMENTO. IV Congresso Brasileiro de Gestão Ambiental, Salvador, BA, v. 9, p.1-5, nov. 2013. Anual.

RAIA JÚNIOR, Archimedes Azevedo (1995). Uma Avaliação do Modelo META para Cálculo de Custos de Transportes e seu Uso na Tributação de Terrenos Urbanos Ociosos. São Carlos, Dissertação (Mestrado). 144p. Escola de Engenharia de São Carlos, Universidade de São Paulo.

SILVA, Antônio Nélson Rodrigues da (1993). O Custo do Solo Urbano Ocioso e Uma Nova Sistemática de Tributação de Propriedade. São Carlos, Tese (Doutorado), 137p. Escola de Engenharia de São Carlos, Universidade de São Paulo.

BORGES, Marília Vicente. O ZONEAMENTO NA CIDADE DO RIO DE JANEIRO: gênese, evolução e aplicação/ Marília Vicente Borges - Rio de Janeiro, RJ, 2007. Dissertação de Mestrado - Universidade Federal do Rio de Janeiro - Instituto de Pesquisa e Planejamento Urbano e Regional da UFRJ 


\section{AUTORES}

ORCID: https://orcid.org/0000-0002-0378-6016

MURILO PEREIRA TREVISAN | Centro de Ensino Superior de Maringá | Engenharia Civil | Maringá, PR. Brasil | Correspondência para: Avenida Higienópolis, $11001^{\circ}$ andar Centro - Londrina - PR | E- maill: murilo.p_trev@hotmail.com

ORCID: https://orcid.org/0000-0002-6558-6378

PAULO ROBERTO NINO JÚNIOR | Centro de Ensino Superior de Maringá | Engenharia Civil | Maringá, PR. Brasil | Correspondência para: Avenida Higienópolis, $11001^{\circ} \mathrm{a}$ dar - Centro - Londrina - PR | E-maill: paulo.robertonino@ gmail.com

\section{COMO CITAR ESTE ARTIGO}

TREVISAN, Murilo Pereira; NINO JÚNIOR, Paulo Roberto. Proposta de Expansão para a Cidade de Nova Esperança- PR. MIX Sustentável, [S.I.], v. 4, n. 3, p. 103-108, out-mar. 2018. ISSN 24473073. Disponível em:<http://www.nexos.ufsc.br/index.php/mixsustentavel>. Acesso em: dia mês. ano. doi:https://doi. org/10.29183/2447-3073.MIX2018.v4.n3.103-108.

DATA DE ENVIO: 15/01/2018 